of climate changes on Mars, the postViking consensus is that the planet never had an atmosphere as thick, or as moist, as that of Earth.

While the bulk of the 232 pages is devoted to surface geology - craters, volcanoes, canyons, chaotic terrain, channels, polar deposits and so on - also included are up-to-date summaries of our knowledge of the planet's interior and atmosphere. The book concludes with a contribution by Harold Klein (one of the biologists on the Viking team) reviewing the results of Viking's search for life, and a short chapter on Phobos and Deimos.

The casual browser will no doubt be seduced by the book's beautiful pictures, but the text is intended for serious readers. I found occasions where the inclusion of a few more key maps would have clarified the discussion, but in general the text is meticulously prepared. The few errors are clearly slips of the pen - for example,
Schiaparelli and not Antoniadi drew canals on Mars in 1877; and it was Mariner 4, and not Mariners 6 and 7, that made the first accurate measurements of atmospheric pressure.

While this is a Viking view of Mars, it would be unfair to call it Carr's view of Mars. The author has gone to great lengths to provide an unbiased description of what is in the pictures and a summary of major current interpretations. A reader looking for a simplistic approach which "explains" everything on Mars may be disappointed occasionally, but the serious student will appreciate Carr's painstaking effort to describe fairly the continuing debate about the many perplexing things that Viking saw and measured. The Surface of Mars is that rare beast, a book about Mars which does justice to its subject.

J. Veverka is at the Laboratory for Planetary Studies, Cornell University, Ithaca, New York.

\title{
The patchwork of plant biochemistry
}

\section{Philip Rubery}

The Biochemistry of Plants: $A$ Comprehensive Treatise. Eight-volume set. Editors-in-chief P.K. Stumpf and E.E. Conn. (Academic: 1980-1982.) Eightvolume set $£ 303.60, \$ 480$. Single volumes also available.

A COMPREHENSIVE treatise on plant biochemistry - some two million words and twenty-five thousand literature citations. What picture of the current state of plant biochemistry emerges from this mountain of paper, and how will future progress in the field be influenced?

Carbohydrate metabolism in its broadest sense -- including photosynthesis, photorespiration, gluconeogenesis, energy storage and utilization, and the biosynthesis of structural polymers - is perhaps the most developed area of plant biochemistry and is well served by several volumes of the Treatise. Here, by and large, there are hard data on specifically botanical systems. However, research in

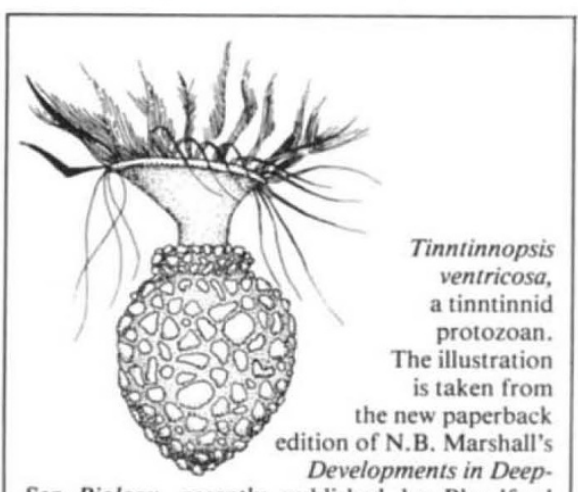

Sea Biology, recently published by Blandford Press, price $\mathbf{8} 8.95$. For review, see Nature 283, 507; 1980. plant biochemistry is notoriously patchy and it is rather depressing to find so many chapters on aspects of primary metabolism which deal almost exclusively with data from animals and microorganisms. This is no fault of the authors (who commonly offer routine apologia), but rather is a consequence of absence of information from plants or, more often, of a hotchpotch of superficial and fragmented investigations from which a connected account cannot be constructed. It is to be hoped that such chapters, which mostly make the best of a bad job, will stimulate efforts to improve our understanding of metabolic processes in plants.

That this need not be an empty comparative exercise is shown by Vol. 4, on lipids, where the rapid progress in unravelling the distinctive features of plant systems is excellently covered. In contrast, much of the volume on proteins and nucleic acids (Vol. 6) is rather disappointing in that it is heavily orientated towards non-plant material in the general chapters on macromolecule biosynthesis. Inevitably parts of this volume will date rapidly and recent progress in plant molecular biology already gives it rather an old-fashioned look. It is noticeable that there is a greater sense of vitality when individual plant topics are dealt with (e.g. proteases and their inhibitors, plant tumours, plant viruses) than when the basic biochemistry is reviewed.

Volume 7 on secondary plant products covers an interesting cross-section of subjects. It reveals, for different classes of compounds, situations ranging from structural information accompanied by biogenetic hypotheses, through tracer studies with little supportive enzymology, to the phenolic compounds where the majority of enzymes in some pathways have been purified and characterized, and the regulation of enzyme levels and of the flux through the pathway have been studied in detail. The highly successful studies of flavonoid biosynthesis from phenylalanine illustrate what is being achieved using modern methods, and show how investigation of what biochemists of other persuasions may regard as obscure compounds can yield information of wide significance.

The Treatise (the first three volumes of which have been reviewed in Nature already - 292, 783;1981) succeeds in a number of ways. It is a valuable work of reference; the individual volumes are well indexed and there are cross-references within and between volumes. It is relatively easy to track down specific topics in spite of the lack of a cumulative index for the work as a whole. In addition the deliberate overlaps - photorespiration, for example, is explicitly covered in Vols 2 (on metabolism and respiration) and 8 (photosynthesis) - means that each volume is self-contained and can be used as a text in its own right.

As an illustration, Vol.5 deals with amino acids (non-protein amino acids are covered in Vol.7). Besides coverage of individual families of compounds, there are chapters on physical and chemical properties, nitrogen fixation, ammonia and sulphate assimilation, transport of nitrogen compounds and the effects of environmental stress on amino acid accumulation.

For beginners, the authoritative accounts of well-investigated topics will facilitate entry into the appropriate fields. On the other side of the coin, the clear signposts to gaps in our knowledge should help identify attractive problems worthy of investigation. For these to be recognized, however, it is important to emphasize the biological context; plant biochemistry (and biophysics) must be seen in relation to the study of plants as well as of molecules. The mutual reinforcement between developmental, physiological and molecular approaches has already been fruitful in many areas. It is perhaps an inevitable weakness that the editors in concentrating on biochemistry per se and employing over one hundred authors, have not been uniformly successful in showing why the biochemistry is worth doing. The first chapters in Vol. 7 do, however, attempt this type of integration in a fairly systematic way.

Overall the Treatise is much to be recommended. It is an unfortunate - and unavoidable - paradox that many of the best chapters which deal with active and expanding topics will also go out of date most rapidly.

Philip Rubery is a Lecturer in Biochemistry at the University of Cambridge. 